\title{
Towards Ultracold Chiral Molecules
}

\author{
Timur A. Isaev ${ }^{a}$ and Robert Berger ${ }^{\star b}$
}

\begin{abstract}
Atoms can be cooled and trapped efficiently with the help of lasers. So-called Doppler cooling takes advantage of momentum transfer upon absorption and emission of photons and of Doppler shifts to facilitate effectively closed optical absorption-emission loops, by which atoms are slowed down and cooled. Due to the wealth of internal degrees of freedom accessible in molecules, it was assumed for a long time that similarly closed optical loops cannot be realised for molecules. After an early theoretical proposal by Di Rosa for diatomic molecules, such cooling has been achieved in this decade for SrF, YO, CaF and YbF. It has been outlined recently that also polyatomic molecules should be coolable with lasers and classes of molecules expected to be amenable to this have been proposed by the present authors. Experimental success in laser cooling of $\mathrm{SrOH}$ has been reported. The status of cooling polyatomic molecules with lasers and the prospects for obtaining ultracold chiral molecules is reviewed herein.
\end{abstract}

Keywords: Chiral molecules · Laser cooling
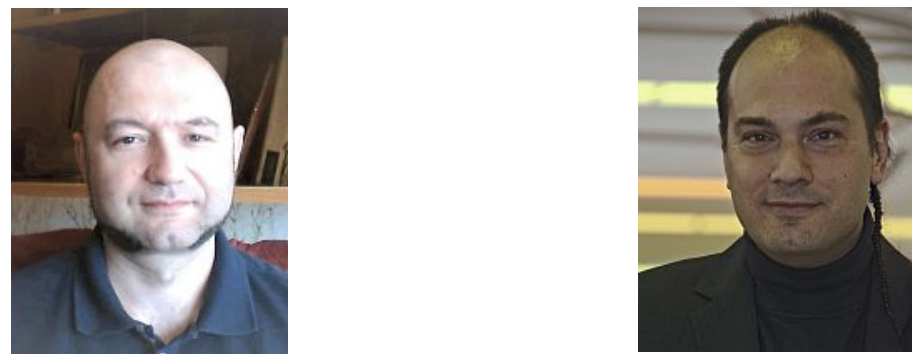

Timur Isaev studied physics at SaintPetersburg State University in Russia. After graduating from SPbSU in 1994 he started working on calculations of the effects of space parity non-conservation in diatomic molecules in the group of Anatoly Titov at Petersburg Nuclear Physics Institute (PNPI). After receiving his PhD Timur Isaev joined the group of Robert Berger at Frankfurt Institute for Advanced Studies as a postdoctoral researcher. In 2015 he returned to Saint-Petersburg, where he was appointed as Senior researcher in NRC 'Kurchatov Institute' - PNPI.

\footnotetext{
${ }^{*}$ Correspondence: Prof. Dr. R. Berger ${ }^{b}$ E-mail: robert.berger@uni-marburg.de aNational Research Center 'Kurchatov Institute' Petersburg Nuclear Physics Institute Orlova Roscha, 1, 188300 Gatchina, Russia

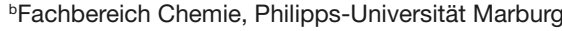
Hans-Meerwein-Straße 4, 35032 Marburg, Germany
}

Robert Berger studied in Berlin and Münster. After receiving his $\mathrm{PhD}$ for his work on theoretical spectroscopy in the group of Martin Klessinger, he spent a postdoctoral stay at ETH Zurich with Martin Quack. He returned with a Liebig fellowship to TU Berlin, where Helmut Schwarz was his mentor. Robert Berger was awarded subsequently a junior research group by the VolkswagenFoundation and became fellow of the Frankfurt Institute for Advanced Studies. After an appointment as associate professor at TU Darmstadt, he became full professor for Theoretical Chemistry at Philipps-Universität Marburg. (Photo: Sebastian Marquardt)

\section{Introduction}

Cold atoms and molecules are perfect objects for performing high-precision spectroscopic measurements and for studying chemical reactions at extremely low energies. In favourable cases, single atoms or molecules can be kept isolated in a trap and then studied under uniquely well-defined conditions. Cooling of atoms with lasers is meanwhile a well-established technique that has its roots in the 1970s. Temperatures down to about hundred microkelvin can be achieved by Doppler cooling ${ }^{[1-5]}$ and subsequent sub-Doppler cooling of atoms ${ }^{[6,7]}$ allows temperature to be lowered even further, for instance such that intriguing phenomena like Bose-Einstein condensation $^{[8-10]}$ can be realized. ${ }^{[11,12]}$

Due to the various internal degrees of freedom available to diatomic molecules, application of laser cooling techniques was for a long time confined to the domain of atomic physics. Di Rosa pointed out in 2004, however, that certain openshell diatomic molecules should allow for Doppler cooling ${ }^{[13]}$ and meanwhile molecules such as SrF, YO, CaF and YbF could be cooled with lasers experimentally. ${ }^{[14-22]}$ We reported recently on the possibilities of laser cooling polyatomic molecules, including also chiral systems, which should give access to cold samples of polyatomic molecules and perfect conditions for precision spectroscopy. ${ }^{[23]}$

In this special issue of CHIMIA devoted to physicochemical aspects of molecular chirality we will review strategies to obtain ultracold atoms and ions, discuss the challenges in obtaining cold molecules, highlight electronic situations that are favourable for cooling molecules with lasers, present classes of molecules for which such favourable conditions can be expected, point to recent developments and outline the perspectives for future research emerging from the availability of ultracold samples of chiral molecules.

\section{Cooling with Lasers}

As opposed to atoms, molecules possess rotational and vibrational degrees of freedom. In the gas phase, these various degrees of freedom are not necessarily in thermal equilibrium so that molecules can be obtained for instance as vibrationally hot, but rotationally cold species, a situa- 
tion frequently encountered in supersonic jet expansions. ${ }^{[24-26]}$ Techniques to cool rovibrational degrees of freedom have been developed (see e.g. refs. [27-29]), so that we focus herein on the challenge to control the translational motion and thereby lower the corresponding temperature.

A rather versatile possibility is to cool an inert gas (buffer gas) such as helium to low temperatures and mix it with the gaseous species of interest (see ref. [30] for a review). Such a buffer gas cooling can give temperatures as low as a few Kelvin.

Even lower temperatures can be achieved with the help of lasers. Photons carry a linear momentum, which due to linear momentum conservation is transferred upon absorption and emission of light to or from the target. The photoelectric effect was described by Einstein within a corpuscular theory for electromagnetic radiation and Compton subsequently invoked the conservation of linear momentum of light particles in order to explain the angle dependent wavelength changes of X-rays in what was subsequently coined Compton scattering. An object absorbing a photon thus experiences a change in linear momentum and recoils subsequently upon emission of a photon. This momentum transfer is exploited in Doppler cooling. If a photon is absorbed from a certain direction and subsequently emitted such that it travels further in the same direction, the net linear momentum transfer vanishes. If photons absorbed stem only from one direction, but are emitted spontaneously in diverse directions, a net momentum transfer results, quite similar to the linear momentum transfer that a wind of air molecules causes on a macroscopic object. The specific trick of Doppler cooling is now ${ }^{[1,2]}$ that photons from a laser source are slightly detuned bathochromically (often referred to as red-detuning) from an atomic or molecular resonance such that photons are not absorbed when the atoms or molecules are at stand-still or move away from the laser source. If the objects travel, however, towards the laser source, photons impinging on the atom or molecule appear in the moving frame of the object shifted to higher frequency due to the Doppler effect and thus can come into resonance with the transition frequency. Thus, photons from only one direction can be absorbed, but later be spontaneously emitted in various directions, which produces the net momentum transfer described above. Typically $10^{4}$ or more photons need to be absorbed and emitted in a closed optical loop to achieve significant translational cooling of the objects. If now six laser beams are spatially arranged such that bathochromically detuned photons impinge from all directions $( \pm x, \pm y$ and $\pm z)$, atoms or molecules that tend to move away from the crossing re- gion of the laser beams are slowed down. The lasers exert a frictional force that slows down the object. But it is not trapped. Trapping can be achieved for instance with the help of additional magnetic fields (magneto-optical trap), or with the help of an electric quadrupole ion trap (Paul trap) when the objects are charged. Frequently trapped species are neutral alkali metal atoms and monocationic ions of the alkaline earth metals. Those have in their electronic ground state an unpaired electron in an $s_{1 / 2}$ orbital over a closed shell from where it can undergo an electric dipole transition to a $p_{1 / 2}$ orbital, at least in this simplified orbital picture. The alkaline earth ions can form so-called Coulomb crystals in the trap, by which other co-stored ions can be cooled sympathetically.

Evidently, closed optical loops are crucial for laser cooling. Any departure from the effective two-level picture leads to more or less severe losses of atoms or ions when no countermeasures are taken. And this is the particular challenge for laser cooling of molecules instead of atoms. Due to the availability of additional rotational and vibrational degrees of freedoms, it is difficult to find an effective two-level system realized as rovibronic transitions can typically take place between diverse levels. In the simplified Franck-Condon (FC) approximation, it is mainly the availability of additional vibrational states that impacts on the efficiency of a laser cooling scheme for molecules (see Fig. 1). But the simple FC picture provides also the guiding principle for finding laser coolable molecules: Those should have similar equilibrium structures and vibrational frequencies in the electronic states involved in the optical loop to be amenable to laser cooling to give favourable FC factors. If the FC factor is $q_{v^{\prime}, v}$ $=\left|\left\langle v^{\prime} \mid v\right\rangle\right|^{2}$ for a given transition between vibrational states with quantum numbers $v^{\prime}$ in the electronically excited state and $v$ in the electronic ground state, then the probability to stay in this loop for $10^{4}$ cycles

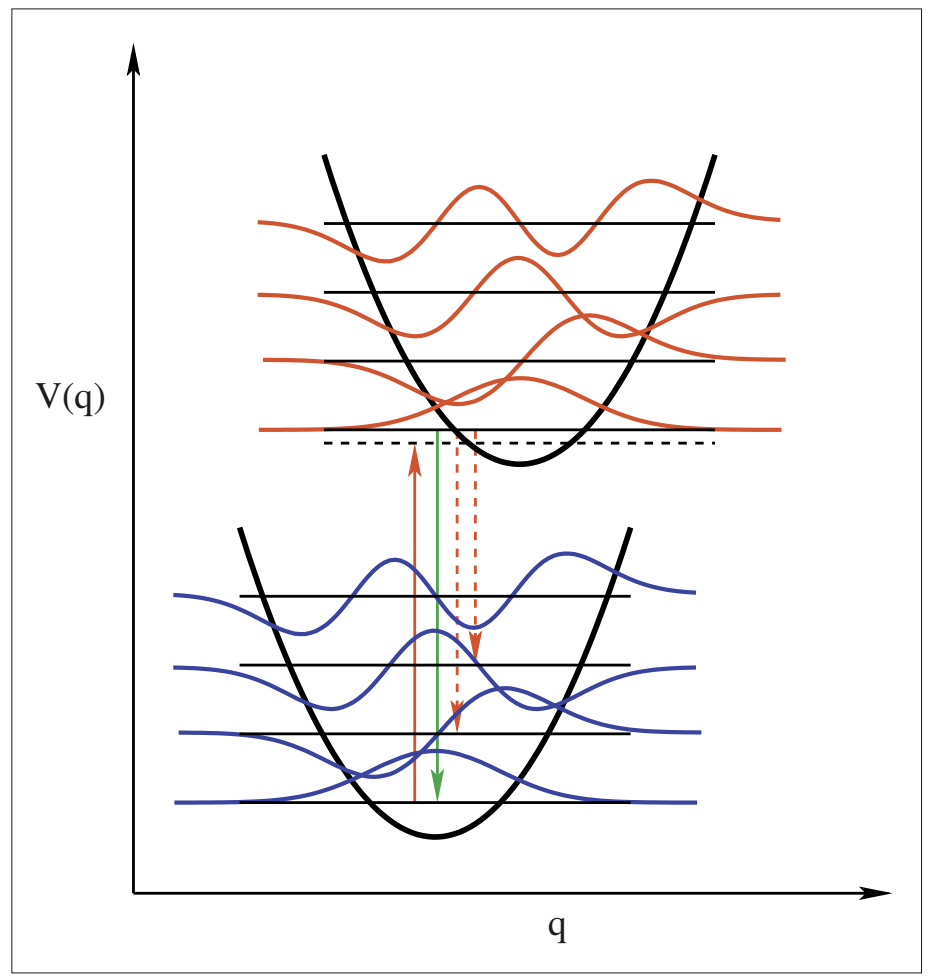

Fig. 1. Laser cooling situation for molecules. Shown are harmonic potentials $V(q)$ of the electronic ground- and excited state as a function of the normal coordinate $q$. Harmonic oscillator levels are sketched as horizontal lines together with corresponding wavefunctions that are shifted to have their zero level lined up with the energy eigenvalues. Lasers are bathochromically detuned as indicated by the red arrow pointing upwards, such that the 0-0'-transition can get into resonance due to the Doppler effect for molecules propagating towards the laser source. Upon spontaneous emission, the system can stay in the optical loop by decaying back to the vibrational ground state $v=0$ (green arrow pointing downwards) or decaying to excited vibrational states $v=1,2, \ldots$ of the electronic ground state (red dashed arrows). For the situation sketched above it is clear that after a few adsorption-emission cycles most molecules would end up in long-lived excited vibrational states of the electronic ground state. 
is $\left(q_{v^{,}, v}\right)^{10^{4}}$. So even if $q=0.9999$ and thus very close to unity, after $10^{4}$ cycles only about one third of the molecules would still be in the same loop. Given this, it was initially assumed to be impossible to Doppler cool molecules.

\section{Classes of Molecules for Laser Cooling}

The quest for laser cooling of molecules was addressed by Di Rosa in 2004, ${ }^{[13]}$ who pointed out three requirements for laser cooling, namely a band system that is strongly allowed in one-photon transitions, a highly diagonal Franck-Condon matrix and no intertwined electronic levels between those selected for optical cycling. He suggested the various diatomic molecules of the alkaline earth monohydride series $(\mathrm{MH}$, with $\mathrm{M}=\mathrm{Be}$, $\mathrm{Mg}, \mathrm{Ca}, \mathrm{Sr}, \mathrm{Ba}$ ) and $\mathrm{AlH}, \mathrm{AlF}$ and $\mathrm{AlCl}$ as candidate systems and worked out the specific laser cooling scheme for calcium monohydride. [13]

We, together with Steven Hoekstra from the University of Groningen, were interested in cold open-shell diatomic molecules to use them as ultra-sensitive probes for fundamental parity-violating interactions (see below). As parity-violating effects are known to increase steeply with increasing nuclear charge, ${ }^{[31-34]}$ we were in particular led to consider polar diatomic molecules with heavy nuclei such as radium monofluoride (RaF), ${ }^{[35]}$ which has also a couple of other favourable features to render it an attractive molecular all-in-one probe for fundamental physics. ${ }^{[36,37]}$

Although we considered specifically the prospects to realise Doppler cooling of $\mathrm{RaF}$, we looked for general criteria as to identify open-shell diatomic molecules with one electron over a closed shell that feature highly diagonal FC matrices. As we pointed out in ref. [35], the situation is favourable if the unpaired electron undergoes transitions between orbitals that do not contribute to the chemical bonding. Then the bonding situation is not changed upon electronic transition, which implies similar equilibrium structures and also nearly parallel potential energy curves, so that highly-diagonal FC matrices can be expected. We pointed out that three classes of such situations appear favourable:[35] i) When the electron is in a lone orbital such that the electron is pointing away from the bonding region, ii) when the electron is essentially in an atom-like orbital, e.g. in a $\mathrm{p}_{\pi}$-orbital in metal hydrides as hydrogen has no energetically low-lying p-orbitals or in $\mathrm{d}_{\delta}$-orbitals, and iii) when the electron is in a rather diffuse orbital, such that overlap with the molecular core is small, a situation frequently found in heavy atom molecules.
When the electronic transition of interest is mostly characterised by transitions between these situations, favourable FC factors for laser cooling can arise. And, indeed, this is what is expected for the ${ }^{2} \prod-{ }^{2} \sum$ transition in $\mathrm{RaF}$.

We turned subsequently to molecules with more than two atoms in the hope to identify even polyatomic candidates for being cooled with lasers. ${ }^{23]}$ Guided by our previous consideration for the electronic situation in diatomic molecules and by the notion that the electron undergoing the transition should be mostly in orbitals that do not affect the chemical bonding situation in the molecules, we considered straightforward substitution of halogens such as fluorine in the aforementioned alkaline earth metal fluorides by pseudohalogens (such as CN, NC, CP, PC, OCN, NCO, $\mathrm{SCN}, \mathrm{NCS}, \mathrm{SeCN}, \mathrm{NCSe}, \mathrm{N}_{3}$ ) or other functional groups (such as $\mathrm{OH}, \mathrm{SH}, \mathrm{CH}_{3}$ ) as for these similar electronic situations might be expected. And indeed, the spectroscopy of some of the candidates $(\mathrm{CaNC}$, $\mathrm{CaOH}, \mathrm{CaCH}_{3}$ and $\mathrm{MgCH}_{3}$ ) is reasonably well explored, so that one could hope for chances to laser cool such molecules, and also our numerical calculations indicated that when assuming two so-called repump lasers, which bring molecules from a higher vibrational state back into the optical loop, $\mathrm{CaNC}$ and $\mathrm{CaOH}$ are promising candidates (see Fig. 2).

The group of Doyle achieved meanwhile laser cooling of SrOH. ${ }^{[38]}$ Whereas we had initially Doppler cooling in mind, the group went for so-called Sisyphus laser cooling, which necessitates a similar electronic situation as Doppler cooling, but requires much fewer photons to be scattered. One of us studied also $\mathrm{RaOH}$ theoretically, ${ }^{[39]}$ which might also serve as a candidate for laser cooling. It has been emphasised in ref. [40] that triatomic molecules such as the metal hydroxides and specifically $\mathrm{YbOH}$ have advantages when studying parity-odd and time-reversal-odd effects such as the electric dipole moment of the electron.

Our focus was in particular on methyl derivatives (see Fig. 3 for the molecular structure and the molecular orbitals that are mostly relevant for the electronic transition), as those could correspond to the first non-linear molecules to be laser cooled and would open up chances to progress towards ultracold chiral molecules: As a first idea in this direction, we suggested to consider the isotopically chiral methyl group CHDT and thus compounds of the type MgCHDT and CaCHDT. ${ }^{[23]}$ Kozyryev et

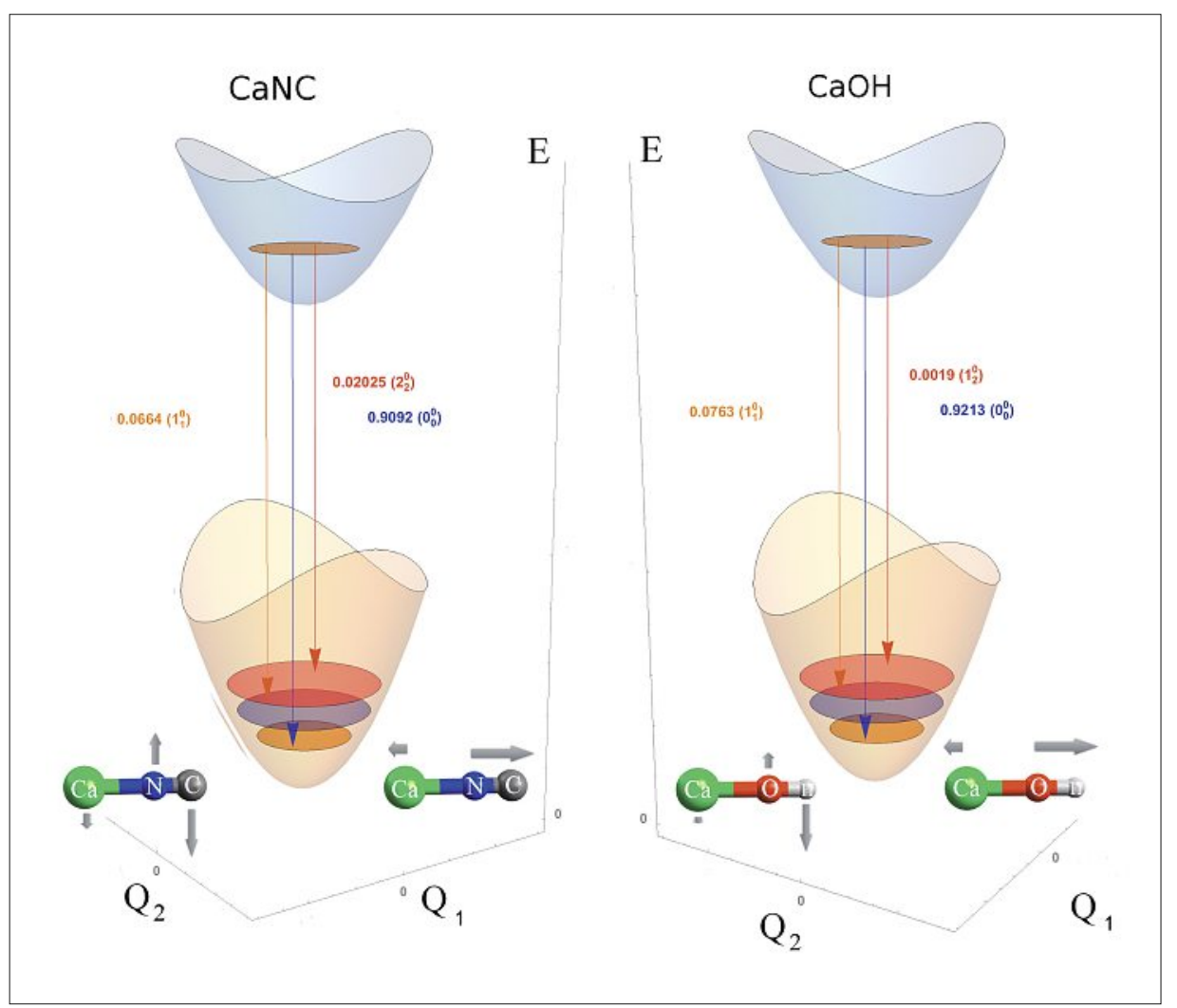

Fig. 2. Schematic representation of potential laser cooling transitions in $\mathrm{CaNC}$ and $\mathrm{CaOH}$. Shown are those transitions with the largest Franck-Condon factors, sketches of a two-dimensional cut through the multi-dimensional Born-Oppenheimer potential energy hypersurfaces and an illustration of normal modes (see ref. [23] for details). Reprinted figure with permission from T. A. Isaev and R. Berger, Phys. Rev. Lett. 2016, 116, 063006. Copyright 2016 by the American Physical Society. 


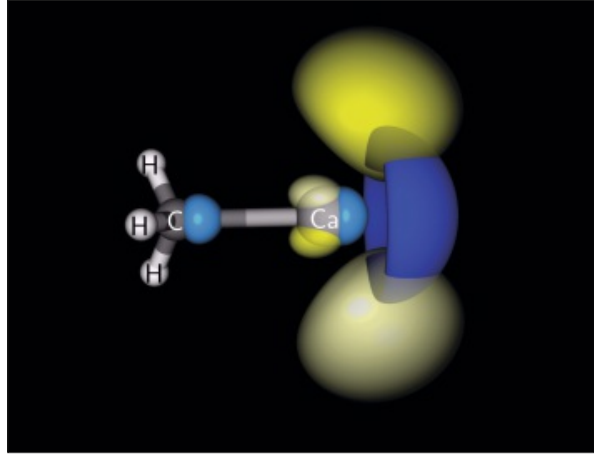

Fig. 3. Sketches of non-bonding, singlyoccupied orbitals involved in potential laser cooling of $\mathrm{CaCH}_{3}$. The electronic ground state orbital is shown in blue, the excited state orbital is shown in yellow (see ref. [23] for details). Reprinted figure with permission from $\mathrm{T}$. A. Isaev and R. Berger, Phys. Rev. Lett. 2016, 116, 063006. Copyright 2016 by the American Physical Society.

al. proposed subsequently to look at alkaline earth alkoxides (MOR) instead, so that the residue $\mathrm{R}$ can be chosen as $\mathrm{CH}_{3}$, CHDT or other alkyl groups with the prospects to reach better cycling rates. ${ }^{[41]}$

\section{Perspectives}

As of yet, laser cooled and trapped chiral molecules have not been reported, but once available one can hope to study fundamental properties of chiral molecules with unprecedented accuracy. Among the various promising perspectives is to attempt a high-resolution measurement of parity violating energy differences either in the frequency domain ${ }^{[42-45]}$ or by observation of quantum beats in time-dependent schemes $^{[46-49]}$ (see also refs. [50-54] for a review).

Other possibilities include the observation of mode-selective tunnelling dynamics of chiral molecules under collision-free conditions, ${ }^{[55,56]}$ light-induced stereomutation of chiral molecules, ${ }^{[57-59]}$ interaction energies between chiral molecules, ultracold chemistry with chiral molecules and corresponding state-to-state resolved reaction cross-section, phase transitions due to competing interaction between chiral molecules in specifically prepared optical lattices and the search for variation of fundamental constants such as the protonelectron mass ratio.

Ultra-cold chiral molecules can thus be expected to become versatile probes for a wealth of intriguing physical and chemical phenomena.

\section{Conclusion}

We have outlined principles of Doppler cooling of atoms and molecules and have highlighted the particular challenges and opportunities for cooling of molecular systems. General features and explicit candidate systems for laser-coolable polyatomic molecules have been reviewed and the prospects to obtain chiral molecules have been discussed.

\section{Acknowledgements}

The authors thank Jürgen Stohner, Martin Quack, Steven Hoekstra, Ronnie Kosloff, Christiane Koch and Kilian Singer for discussions. RB acknowledges support from Deutsche Forschungsgemeinschaft via Sonderforschungsbereich 1319 (ELCH). TI is grateful for support to RSF fund 18-12-00227.

Received: May 15, 2018

[1] T. W. Hänsch, A. L. Schawlow, Opt. Commun. 1975, 13, 68 .

[2] D. J. Wineland and H. Dehmelt, Bull. Am. Phys Soc. 1975, 20, 637.

[3] D. J. Wineland, R. E. Drullinger, F. L. Walls, Phys. Rev. Lett. 1978, 40, 1639.

[4] W. Neuhauser, M. Hohenstatt, P. Toschek, H. Dehmelt, Phys. Rev. Lett. 1978, 41, 233.

[5] S. Chu, L. Hollberg, J. E. Bjorkholm, A. Cable, A. Ashkin, Phys. Rev. Lett. 1985, 55, 48.

[6] A. Aspect, E. Arimondo, R. Kaiser, N Vansteenkiste, C. Cohen-Tannoudji, Phys. Rev. Lett. 1988, 61, 826 .

[7] P. D. Lett, R. N. Watts, C. I. Westbrook, W. D Phillips, P. L. Gould, H. J. Metcalf, Phys. Rev. Lett. 1988, 61, 169.

[8] S. N. Bose, Z. Phys. 1924, 26, 178.

[9] A. Einstein, Sitzungsber. Kgl. Preuss. Akad. Wiss. 1924, 22, 261.

[10] A. Einstein, Sitzungsber. Kgl. Preuss. Akad. Wiss. 1925, 23, 3.

[11] M. H. Anderson, J. R. Ensher, M. R. Matthews, C. E. Wieman, E. A. Cornell, Science 1995, 269, 198.

[12] K. B. Davis, M. O. Mewes, M. R. Andrews, N. J. van Druten, D. S. Durfee, D. M. Kurn, W. Ketterle, Phys. Rev. Lett. 1995, 75, 3969

[13] M. D. DiRosa, Eur. Phys. J. D 2004, 31, 395.

[14] E. S. Shuman, J. F. Barry, D. DeMille, Nature 2010, 467,820 .

[15] J. F. Barry, E. S. Shuman, E. B. Norrgard, D. DeMille, Phys. Rev. Lett. 2012, 108, 103002.

[16] J. F. Barry, D. J. McCarron, E. B. Norrgard, M. H. Steinecker, D. DeMille, Nature 2014, 512 , 286.

[17] M. T. Hummon, M. Yeo, B. K. Stuhl, A. L. Collopy, Y.Xia, J. Ye, Phys. Rev. Lett. 2013, 110 143001

[18] M. Yeo, M. T. Hummon, A. L. Collopy, B. Yan, B Hemmerling, E. Chae, J. M. Doyle, J. Ye, Phys. Rev. Lett. 2015, 114, 223003

[19] V. Zhelyazkova, A. Cournol, T. E. Wall, A. Matsushima, J. J. Hudson, E. A. Hinds, M R. Tarbutt, B. E. Sauer, Phys. Rev. A 2014, 89 , 053416.

[20] B. Hemmerling, E. Chae, A. Ravi, L. Anderegg, G. K. Drayna, N. R. Hutzler, A. L. Collopy, J. Ye, W. Ketterle, J. M. Doyle, J. Phys. B 2016, 49, 174001

[21] S. Truppe, H. J. Williams, M. Hambach, L. Caldwell, N. J. Fitch, E. A. Hinds, B. E. Sauer, M. R. Tarbutt, Nat. Phys. 2017, 13, 1173.
[22] J. Lim, J. R. Almond, M. A. Trigatzis, J. A. Devlin, N. J. Fitch, B. E. Sauer, M. R. Tarbutt, E.A. Hinds, Phys. Rev. Lett. 2018, 120, 123201.

[23] T.A. Isaev, R. Berger, Phys. Rev. Lett. 2016, 116, 063006.

[24] A. Kantrowitz, J. Grey, Rev. Sci. Instr. 1951, 22 , 328.

[25] O. Hagena, W. Henkes, Z. Naturforsch. A 1960, 15,851 .

[26] M. P. Sinha, A. Schultz, R. N. Zare, J. Chem. Phys. 1973, 58, 49.

[27] M. Viteau, A. Chotia, M. Allegrini, N. Bouloufa, O. Dulieu, D. Comparat, P.Pillet, Science 2008 321, 232.

[28] D. M. Reich, C. P.Koch, New J. Phys. 2013, 15, 125028.

[29] M. Hamamda, P.Pillet, H. Lignier, D. Comparat, J. Phys. B 2015, 48, 182001.

[30] N. R. Hutzler, H.-I. Lu, J. M. Doyle, Chem. Rev. 2012, 112, 4803.

[31] M. A. Bouchiat and C. Bouchiat, J. Phys. (Paris) 1974, 35, 899

[32] O. P. Sushkov and V. V. Flambaum, Sov. Phys. JETP 1978, 48, 608.

[33] M. G. Kozlov, Sov. Phys. JETP 1985, 62, 1114.

[34] M. G. Kozlov and L. N. Labzowsky, J. Phys. B 1995, 28, 1933

[35] T. A. Isaev, S. Hoekstra, R. Berger, Phys. Rev. A 2010, $82,052521$.

[36] T. A. Isaev and R. Berger, ArXiv e-prints, 2013, arXiv:1302.5682 [physics.chem-ph].

[37] A. D. Kudashov, A. N. Petrov, L. V. Skripnikov, N. S. Mosyagin, T. A. Isaev, R. Berger, A. V. Titov, Phys. Rev. A 2014, 90, 052513.

[38] I. Kozyryev, L. Baum, K. Matsuda, B. L. Augenbraun, L. Anderegg, A. P. Sedlack, J. M. Doyle, Phys. Rev. Lett. 2017, 118, 173201.

[39] T. A. Isaev, A. V.Zaitsevskii, E. Eliav, J. Phys. $B$ 2017, 50, 225101.

[40] I. Kozyryev, N. R. Hutzler, Phys. Rev. Lett. 2017, $119,133002$.

[41] I. Kozyryev, L. Baum, K. Matsuda, J. M. Doyle, ChemPhysChem 2016, 17, 3641.

[42] O. N. Kompanets, A. R. Kukudzhanov, V. S. Letokhov, L. L. Gervits, Opt. Commun. 1976, $19,414$.

[43] E. Arimondo, P.Glorieux, T.Oka, Opt. Commun. $1977,23,369$

[44] A. Bauder, A. Beil, D. Luckhaus, F. Müller, M. Quack, J. Chem. Phys. 1997, 106, 7558

[45] C. Daussy, T. Marrel, A. Amy-Klein, C. T Nguyen, C. J. Bordé, C. Chardonnet, Phys. Rev. Lett. 1999, 83, 1554.

[46] R. A. Harris, L. Stodolski, Phys. Lett. B 1978, $78,313$.

[47] M. Quack, Chem. Phys. Lett. 1986, 132, 147.

[48] M. Quack, Faraday Discuss. 1994, 99, 389.

[49] R. Berger, Phys. Chem. Chem. Phys. 2003, 5, 12.

[50] M. Quack, Angew. Chem. Int. Ed. 1989, 28, 571.

[51] M. Quack, Angew. Chem. Int. Ed. 2002, 41, 4618.

[52] R. Berger, in 'Relativistic Electronic Structure Theory, Part: 2, Applications', Ed. P. Schwerdtfeger, Elsevier, Netherlands, 2004, Chap. 4, pp. 188-288.

[53] J. Crassous, C. Chardonnet, T. Saue, P. Schwerdtfeger, Org. Biomol. Chem. 2005, 3 , 2218.

[54] M. Quack, J. Stohner, M. Willeke, Annu. Rev. Phys. Chem. 2008, 59, 741 .

[55] M. Gottselig, D. Luckhaus, M. Quack, J. Stohner, M. Willeke, Helv. Chim. Acta 2001, 84, 1846

[56] M. Gottselig, M. Quack, J. Stohner, M. Willeke, Int. J. Mass. Spectr. 2004, 233, 373.

[57] R. Marquardt, M. Quack, Z. Phys. D 1996, 36, 229.

[58] Y. Fujimura, L. González, K. Hoki, D. Kröner, J. Manz, Y Ohtsuki, Angew. Chem. Int. Ed. 2000, 24,4586 .

[59] M. Shapiro, E. Frishman, P. Brumer, Phys. Rev. Lett. 2001, 84, 1669. 